\title{
Exposure of vital cells to necrotic cell lysates induce the IRE1 $\alpha$ branch of the unfolded protein response and cell proliferation
}

\author{
Philipp Rohne $^{1}$ (D) Steven Wolf ${ }^{1,2} \cdot$ Carolin Dörr $^{1} \cdot$ Julia Ringen $^{1} \cdot$ Andrew Holtz $^{1}$ • \\ René Gollan $^{3}$ • Benjamin Renner ${ }^{1}$ - Hans Prochnow ${ }^{1,4} \cdot$ Markus Baiersdörfer $^{1}$. \\ Claudia Koch-Brandt ${ }^{1}$
}

Received: 29 March 2017 /Revised: 15 May 2017 / Accepted: 16 June 2017 / Published online: 7 July 2017

(C) Cell Stress Society International 2017

\begin{abstract}
Necrosis is a form of cell death that is detrimental to the affected tissue because the cell ruptures and releases its content (reactive oxygen species among others) into the extracellular space. Clusterin (CLU), a cytoprotective extracellular chaperone has been shown to be upregulated in the face of necrosis. We here show that in addition to CLU upregulation, necrotic cell lysates induce JNK/SAPK signaling, the IRE1 $\alpha$ branch of the unfolded protein response (UPR), the MAPK/ERK1/2, and the mTOR signaling pathways and results in an enhanced proliferation of the vital surrounding cells. We name this novel response mechanism: Necrosisinduced Proliferation (NiP).
\end{abstract}

The original version of this article was revised.

Electronic supplementary material The online version of this article (doi:10.1007/s12192-017-0825-6) contains supplementary material, which is available to authorized users.

Philipp Rohne

philipp.rohne@googlemail.com

Steven Wolf

wolfs@uchicago.edu

Carolin Dörr

cdoerr@students.uni-mainz.de

Julia Ringen

jringen@students.uni-mainz.de

Andrew Holtz

andrew.holtz94@gmail.com

René Gollan

renegollan@gmail.com

Benjamin Renner

berenner@students.uni-mainz.de
Keywords Necrosis $\cdot$ Clusterin $\cdot$ UPR $\cdot$ IRE1a $\cdot$ ERK . mTOR

\section{Introduction}

Although the mechanisms of cellular apoptosis are wellknown, the impact of necrotic cell death remains uncertain and is the topic of intense debate. More specifically, little is known about the consequences in neighboring cells of necrotizing tissue. Traditionally, necrotic cell death is defined as uncontrolled cell death stimulated by spontaneous and

Hans Prochnow

hansproc@googlemail.com

Markus Baiersdörfer

mbaiersdoerfer@gmx.de

Claudia Koch-Brandt

koch@uni-mainz.de

Institute of Pharmacy and Biochemistry - Therapeutical Life Sciences, Johannes Gutenberg University of Mainz, Johann-Joachim Becherweg 30, 55128 Mainz, Germany

2 Department of Pathology, The University of Chicago, Chicago, IL, USA

3 Department of Neurology, University Medical Center Mainz, Johannes Gutenberg University Mainz, Mainz, Germany

4 Department of Chemical Biology, Helmholtz Centre for Infection Research GmbH, Braunschweig, Germany 
devastating stressors such as intoxication, injury, or heat (Vanden Berghe et al. 2014). More recently however, necrosis has been reevaluated through detailed observations in various pathologies and diseases. Among others, necrotic cell death can be observed when blood flow is reestablished after instances of low oxygen and nutrient supply, such as after stroke or organ transplantations, known as the ischemia reperfusion injury (IRI) (Kalyanaraman 2013; Yuan 2009). Further, necrotic cell death has been observed to occur in endothelial cells of atherosclerotic plaques (Martinet et al. 2011) and during viral and bacterial infections (Festjens et al. 2006). Likewise, it becomes more and more apparent that necrosis and necrotic cell death mechanisms are underlying features of carcinogenesis, cancer progression, and invasion (Leek et al. 1999; Srikrishna and Freeze 2009; Vakkila and Lotze 2004). Thus, it is hypothesized that cellular necrosis evolved from an uncontrolled accidental cell death mechanism to a welldefined cellular program, regulated by a growing network of cellular modulators (Vanden Berghe et al. 2014).

Intriguingly, one protein that repeatedly surfaced as being upregulated in necrotic tissue, as well as shown to be an indispensable protective tool for the reconstruction of necrotic or damaged tissue is the extracellular glycoprotein clusterin (CLU), also known as apolipoprotein J (APOJ) (Rohne et al. 2016). Secretory clusterin (sCLU) is today the best-defined molecular chaperone acting outside of the cell (Rohne et al. 2016; Wyatt et al. 2012). The processing and maturation of sCLU lead from a single chain protein to a highly glycosylated protein cleaved in two chains with four to five disulfide bonds. Recently, it was reported that the chaperone activity of sCLU is dependent on posttranslational maturation, such as core glycosylation and the proteolytic cleavage. These are prerequisites required for the protein to act in a reducing environment, such as in necrotic tissue (Rohne et al. 2014). By examining the influence of necrotic cell lysates, it was demonstrated that sCLU is upregulated and secreted in vital neighboring cells, which renders sCLU a potential marker for ongoing necrotic cell death in tissues (Bach et al. 2001; Baiersdorfer et al. 2010; Bartl et al. 2001; Rohne et al. 2016; Schwarz et al. 2008; Zhou et al. 2015). Moreover, it was shown that sCLU acts as a scavenging protein in the clearance of misfolded proteins or toxic proteins from the extracellular space, which is mediated by receptors of the LDL receptor gene family, DC-SIGN, and scavenger receptors (Bartl et al. 2001; Leeb et al. 2014; Merlotti et al. 2015; Sabatte et al. 2011; Wyatt et al. 2011; Zlokovic et al. 1996).

In this context, it was shown that sCLU acts as a key factor in sealing wounds and in tissue regeneration after damage, observed at the ocular surface and in the kidney (Bauskar et al. 2015; Nguan et al. 2014). Additionally, sCLU was described as a protective molecule by preventing further damage after myocardial infarction and in other inflammation-related pathologies (McLaughlin et al. 2000; Rohne et al. 2016).
Furthermore, an upregulation and secretion of CLU by axons have been witnessed in response to acute heat trauma and in astrocytes of old head injuries (Troakes et al. 2016). Even though there exists an abundance of studies advocating for a beneficial role of CLU in survival and regeneration of tissues and cells, a defined mechanism is still unknown. Promising results recently published by Martin Gleave and colleagues showed that CLU is subject to BiP-mediated retrotranslocation from the endoplasmic reticulum (ER) to the cytosol ( $\mathrm{Li}$ et al. 2013). Furthermore, they show that intracellular CLU (iCLU) is feasible to promote autophagy by mediating LC3 lipidation (Zhang et al. 2014). More recently, it was demonstrated that a CLU knockout in renal epithelial cells lowers LC3 lipidation, cell viability, and IRE1 $\alpha$ phosphorylation under hypoxic conditions (Alnasser et al. 2016), which are the main cause for IRI and consequent necrosis (Kalyanaraman 2013).

The receptor IRE1 $\alpha$ is part of the unfolded protein response (UPR), a stress activated cellular quality control program residing in the endoplasmic reticulum (ER) that ensures successful folding of ER-resident proteins (Gardner et al. 2013; Ron and Walter 2007). UPR and autophagy are closely related and represent a molecular switch that determines whether a cell is destined for cell death or survival (Hetz 2012; Yan et al. 2015). One side branch of the IRE1 $\alpha$ signaling pathway is the JNK pathway, which leads to the activation of the stress-induced transcription factor AP-1 (Hetz 2012). Indeed, the $c l u$ gene promoter bears AP-1 responsive sites (Michel et al. 1997) that might correlate with IRE1 $\alpha$ activation suggesting a potential interaction of the ER and the extracellular proteostatic system.

In this study, we set out to analyze in addition to the upregulation of CLU by necrotic cell lysates in affected cells if other cytoprotective processes are induced in vital surrounding cells of affected tissue. We show here that necrotic cell lysates specifically induce the IRE $1 \alpha$ branch of the UPR. We further show that in vital cells necrotic cell lysates trigger a proliferative stimulus, which is mediated by ERK $1 / 2$ and mTOR. This phenomenon demonstrates a novel Necrosis-induced Proliferation (NiP) mechanism.

\section{Material and methods}

\section{Cell culture}

HEK-293 cells were grown in the presence of $10 \%$ FBS (Sigma) at $37{ }^{\circ} \mathrm{C}$ in a humidified atmosphere with $5 \% \mathrm{CO}_{2}$. For Western blot, RT- and qRT-PCR experiments $1.5^{*} 10^{6}$ HEK-293 cells were seeded into 6-well plates and grown for at least $20 \mathrm{~h}$. They were subsequently washed once with PBS and set on serum-free media for $4 \mathrm{~h}$ in the presence of DMSO (Roth), Kira6 (Merck), or Parthenolide (Sigma). SP600125 (Sigma) was applied as indicated in the corresponding figure 
legends. After incubation in serum-free media, the cells were stimulated with necrotic cell lysates (see below), human TNF (Sigma), endotoxin-free BSA (Roth), LPS from Salmonella minnesota (Alexis), thapsigargin (Sigma), with or without inhibitors, or DMSO for various times.

\section{Molecular cloning and transfection}

Constructs used were described previously (Prochnow et al. 2013) or were cloned with primers (see Table A2) using the In-Fusion HD Cloning Kit (Clontech Laboratories, Inc.). Transfection of cells were carried out using Turbofect (Thermo Scientific) according the manufacturer's protocol.

\section{Generation of a stable clusterin knockdown}

HEK-293 cells were transfected with pTER-EGFP containing either clusterin knockdown oligonucleotides (shCLU) or scrambled oligonucleotides (Scr) (see Table A2). To receive stable clones, the cells were selected by using Zeocin ${ }^{\mathrm{TM}}$ (Invitrogen).

\section{Preparation and treatment of necrotic cell lysates}

HEK-293 cells were grown in T175 culture flasks (Greiner bio one) to full confluency, removed by trypsin digestion, diluted in serum-free medium, and centrifuged $500 \times \mathrm{g}$ at room temperature for $20 \mathrm{~min}$. The supernatant was discarded and the cells were diluted in fresh serum-free medium or phosphate buffer $\mathrm{pH} 8$ and underwent four freeze/thaw cycles in liquid nitrogen. The lysed cells were then centrifuged at $20,000 \times g$ for $30 \mathrm{~min}$ at $4{ }^{\circ} \mathrm{C}$ and the supernatant was used for stimulation of vital cells.

\section{Cell lysate preparation and Western blotting}

After a time of sensitive incubation, cells were lysed in icecold lysis buffer (50 mM Tris/HCl [pH 8], $150 \mathrm{mM} \mathrm{NaCl}, 1 \%$ $(v / v)$ Triton ${ } \mathrm{X}-100)$ containing phosphatase inhibitor cocktail $\mathrm{V}$ (Calbiochem) and protease inhibitor (Complete mini, Roche). For Western blot analyses 50-100 $\mu$ g protein lysates or $10-30 \mu \mathrm{L}$ cell culture supernatants were denatured and subjected to reducing SDS-PAGE and blotted onto nitrocellulose membranes. For the detection of various proteins, antibodies were used as listed in Table A3. Respective bands were visualized with HRP-linked secondary antibodies.

\section{Synthesis of cDNA and RT- and qRT-PCR}

Stimulated cells were pelleted and total cellular mRNA was isolated using the innuPREP RNA Mini Kit (Analytik Jena). After quantification, the cellular mRNA was reverse transcribed with RevertAid Reverse Transcriptase (Thermo
Scientific). Semi-quantitative RT-PCR analyses were carried out with Maxima Hot Start Green PCR Master Mix (Thermo Scientific) using DNA oligomers as listed in Table A1 or were described previously (Yoshida et al. 2001). All RT-PCRs were applied on 2\% agarose gels, except XBP1 (4\%). Quantitative RT-PCRs were performed using $25 \mathrm{ng} / \mu$ l of oligo dT-reverse transcribed mRNA and KAPA SYBR Fast qPCR Master Mix Rox low (peqlab) in a 7500 Real-Time PCR Instrument (Applied Biosience). Analyses were performed in four replicates using the 7500 Fast System and SDS Software (Applied Biosystems). The DNA oligomers for qRT-PCR analyses are listed in Table A2 or were described previously (Hirota et al. 2006; Sidrauski et al. 2013; van Schadewijk et al. 2012).

\section{AP-1 luciferase reporter assay}

Three-hundred thousand HEK-293 cells were cultivated in 24-well plates for $24 \mathrm{~h}$ and were co-transfected using $1 \mu \mathrm{g}$ pAP-1-Luc (see Table A2) or pTAL-Luc (Clonetech) and $0.4 \mu \mathrm{g}$ pTER-EGFP (Clonetech) for $6 \mathrm{~h}$. Subsequently, the cells were treated as indicated in Fig. 2b. At last, the cells were washed once with PBS and the cell's luciferase activity was

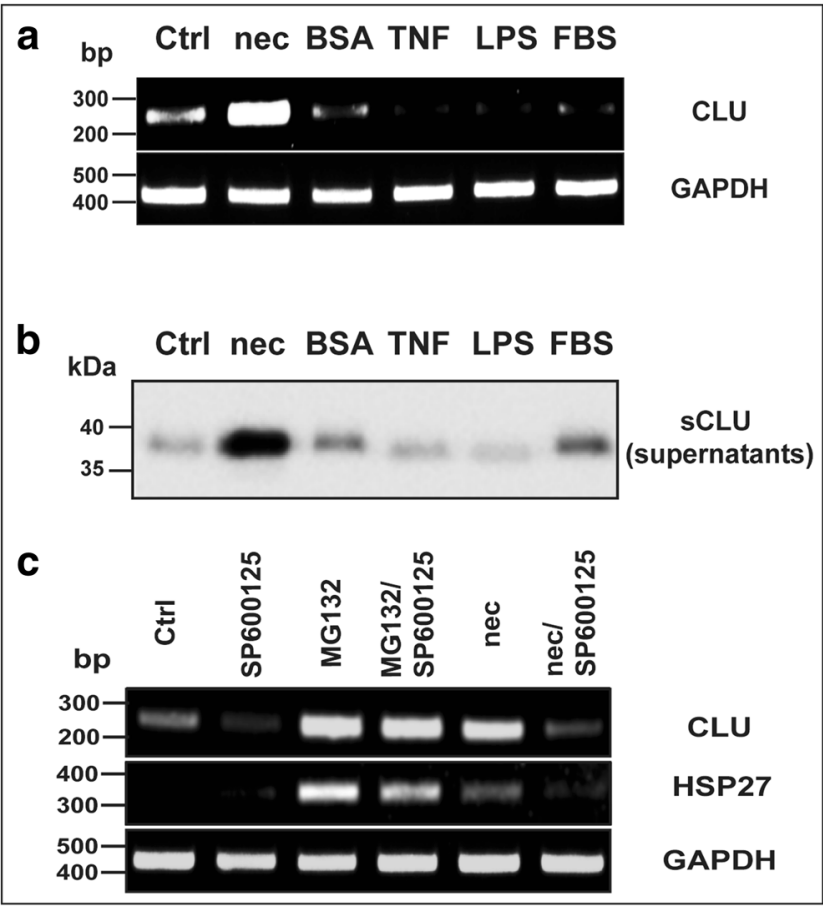

Fig. 1 Necrotic cell lysates induce the JNK/SAPK signaling pathway. HEK-293 cells were stimulated with $1 \mathrm{mg} / \mathrm{mL}$ necrotic cell lysates (nec), BSA, FBS, $5 \mathrm{ng} / \mathrm{mL}$ TNF, or $1 \mu \mathrm{g} / \mathrm{mL}$ LPS. a RT-PCR analyses for CLU transcription with GAPDH as control. b Western blot analyses for secreted sCLU in cell culture supernatants were performed after $6 \mathrm{~h}$ of stimulation. c HEK-293 cells were preincubated with or without $10 \mu \mathrm{M}$ JNK inhibitor SP600125 for $9 \mathrm{~h}$. After preincubation, cells were stimulated with either $10 \mu \mathrm{M}$ MG-132 or $1 \mathrm{mg} / \mathrm{mL}$ necrotic cell lysates (nec) in the presence or absence of SP600125 for $6 \mathrm{~h}$. All experiments were performed at least three times 
measured in white plates using the Luciferase Assay System (Promega) according to the manufacturer's protocol and the FLUOstar Omega luminometer (BMG Labtech). Values were expressed relative to the fluorescence intensity of the respective cell lysates.

\section{Flow cytometer measurements}

To elucidate the relative apoptosis rate, FACS analyses were performed with 500,000 HEK-293 cells plated in 6-well plates and grown for $24 \mathrm{~h}$. The cells were subsequently washed once with PBS and stimulated with necrotic cell lysates for $24 \mathrm{~h}$.
The cells were then removed with trypsin and stained using the PE Annexin V Apoptosis Kit I (BD Bioscience) according the manufacturer's protocol. The samples were measured using a BD FACS Canto II (BD Bioscience) flow cytometer.

\section{Cell viability assays (MTS assays)}

To monitor the viability of HEK-293 cells, (if not indicated otherwise in the figure legends) 5000 cells in $100 \mu \mathrm{L}$ full medium (10\% FBS) were plated into sterile 96-well plates and grown for $24 \mathrm{~h}$. After $4 \mathrm{~h}$ on serum-free, the cells were stimulated with necrotic cell lysates (see below) for 12-24 h.

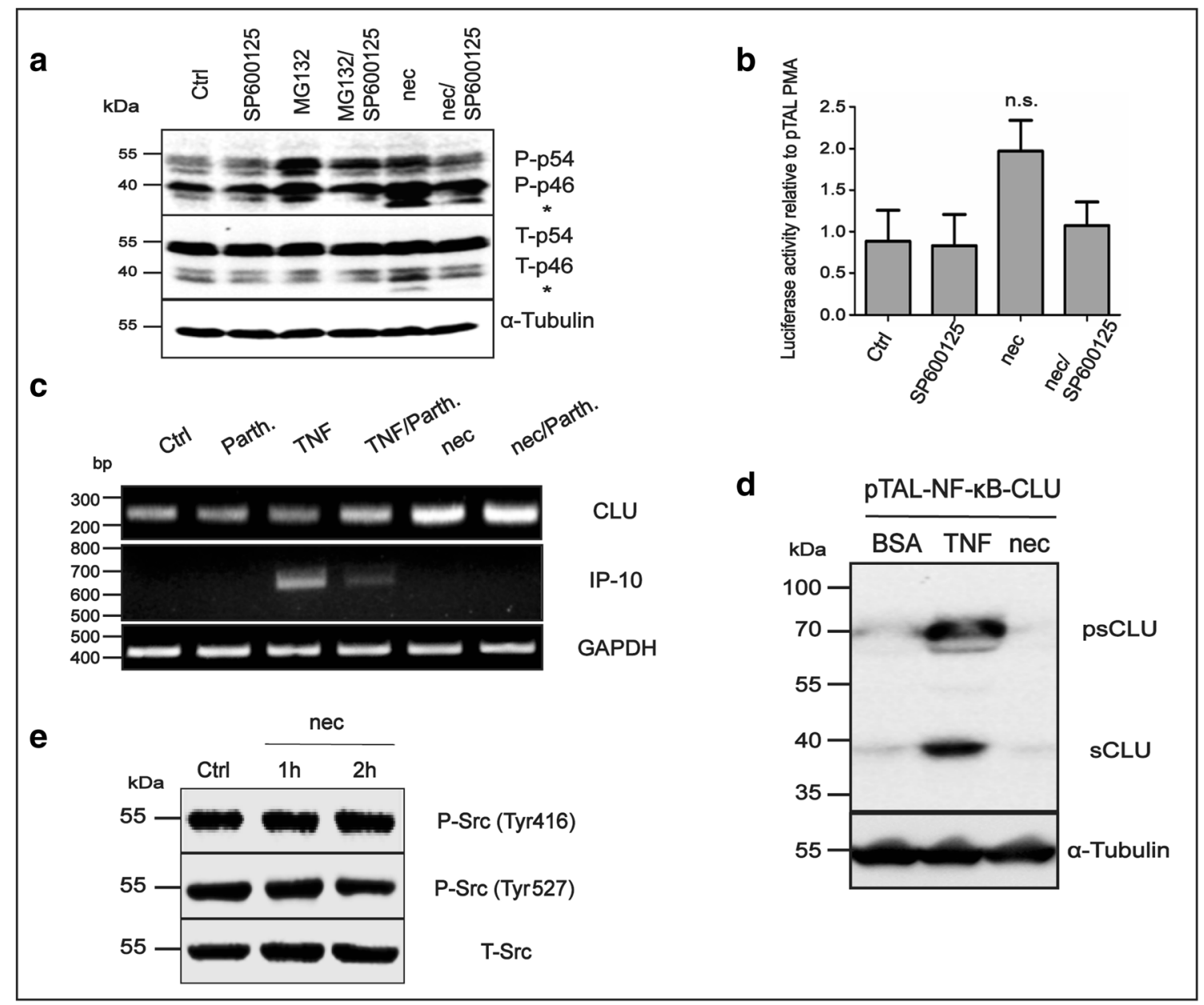

Fig. 2 JNK phosphorylation and AP-1 induction but not NF- $\mathrm{kB}$ signaling are promoted by necrotic cell lysates. (a and b) HEK-293 cells were preincubated with or without $10 \mu \mathrm{M}$ JNK inhibitor SP600125 for $9 \mathrm{~h}$. After preincubation, cells were stimulated with either $10 \mu \mathrm{M}$ MG-132 or $1 \mathrm{mg} / \mathrm{mL}$ necrotic cell lysates (nec) in the presence or absence of SP600125 for $2 \mathrm{~h}$ (Western blot) or $5 \mathrm{~h}$ (reporter assays). a JNK phosphorylation analyses were carried out by Western blot $(n=3)$. b AP-1 luciferase reporter assays: Chemiluminescence was measured and is shown relative to blank vector stimulated with $1 \mu \mathrm{M}$ PMA. As transfection control pTER-EGFP was cotransfected. Data shown are the mean \pm SE of three independent experiments. Data were analyzed by using one-way ANOVA, followed by Tukey's multiple comparison test. c RT-PCR analyses for the transcription of CLU, IP-10 (NF-kB positive control) with GAPDH as loading control were performed in HEK-293 cells preincubated with or without $25 \mu \mathrm{M}$ NF- $\mathrm{KB}$ inhibitor parthenolide (Parth.) for $4 \mathrm{~h}$. After preincubation, cells were stimulated with either $5 \mathrm{ng} / \mathrm{mL}$ TNF or $1 \mathrm{mg} / \mathrm{mL}$ necrotic cell lysates (nec) in the presence or absence of $25 \mu \mathrm{M}$ Parthenolide for $6 \mathrm{~h}(n=2)$. d pTAL-NF-kB-CLU reporter (see Table A2) was transfected into HEK-293 cells for $6 \mathrm{~h}$ in 6well plates. Cells were subsequently plated 1:3 and grown for another $36 \mathrm{~h}$. After $4 \mathrm{~h}$ of serum-free incubation, the cells were stimulated with $5 \mathrm{ng} / \mathrm{mL}$ TNF or $0.5 \mathrm{mg} / \mathrm{mL}$ necrotic cell lysates (nec) for another $6 \mathrm{~h}$. psCLU indicates presecretory uncleaved clusterin. Western blot analyses were carried out using anti-V5 antibody (Life Technologies) $(n=3)$. e Western blot analyses of HEK-293 cells stimulated with $1 \mathrm{mg} / \mathrm{mL}$ necrotic cell lysates (nec) for the indicated times $(n=3)$ 
The medium was then replaced by $50 \mu \mathrm{L}$ fresh serum-free medium and $10 \mu \mathrm{L}$ Cell Titer 96 Aqueous one solution (Promega) was added. The reduction of cell titer solution was measured (490 and $630 \mathrm{~nm}$ ) in an ELISA reader (PowerWave XS, BioTek) within $4 \mathrm{~h}$. In the case of transfection with plasmid DNA or usage of Q-VD-OPh (Sigma), 10,000 HEK-293 cells were seeded and the assays were carried out as described in the corresponding figure legends.

\section{Cell proliferation assays}

One-hundred thousand HEK-cells were seeded into 24-well plates and grown for $24 \mathrm{~h}$. Subsequently, the cells were stimulated with $1 \mathrm{mg} / \mathrm{mL}$ necrotic cell lysates. After $24 \mathrm{~h}$ of incubation in the absence or presence of necrotic cell lysates, cells were trypsinized and total cell numbers were directly determined using a Neubauer counting chamber. In addition, cell proliferation was assayed using the Edu-click system. Briefly, Lumox ${ }^{\circledR} 96-$ well plates (Sarstedt) were coated with Poly-L-lysine (Sigma) and 40,000 HEK-293 cells per well were seeded and grown for $24 \mathrm{~h}$. Subsequently, the cells were stimulated with $1 \mathrm{mg} / \mathrm{mL}$ necrotic cell lysates for another $24 \mathrm{~h}$. Finally, cell proliferation was measured by using the EdU HTS kit (Baseclick) according to the manufacturer's protocol and the FLUOstar Omega luminometer (BMG Labtech).

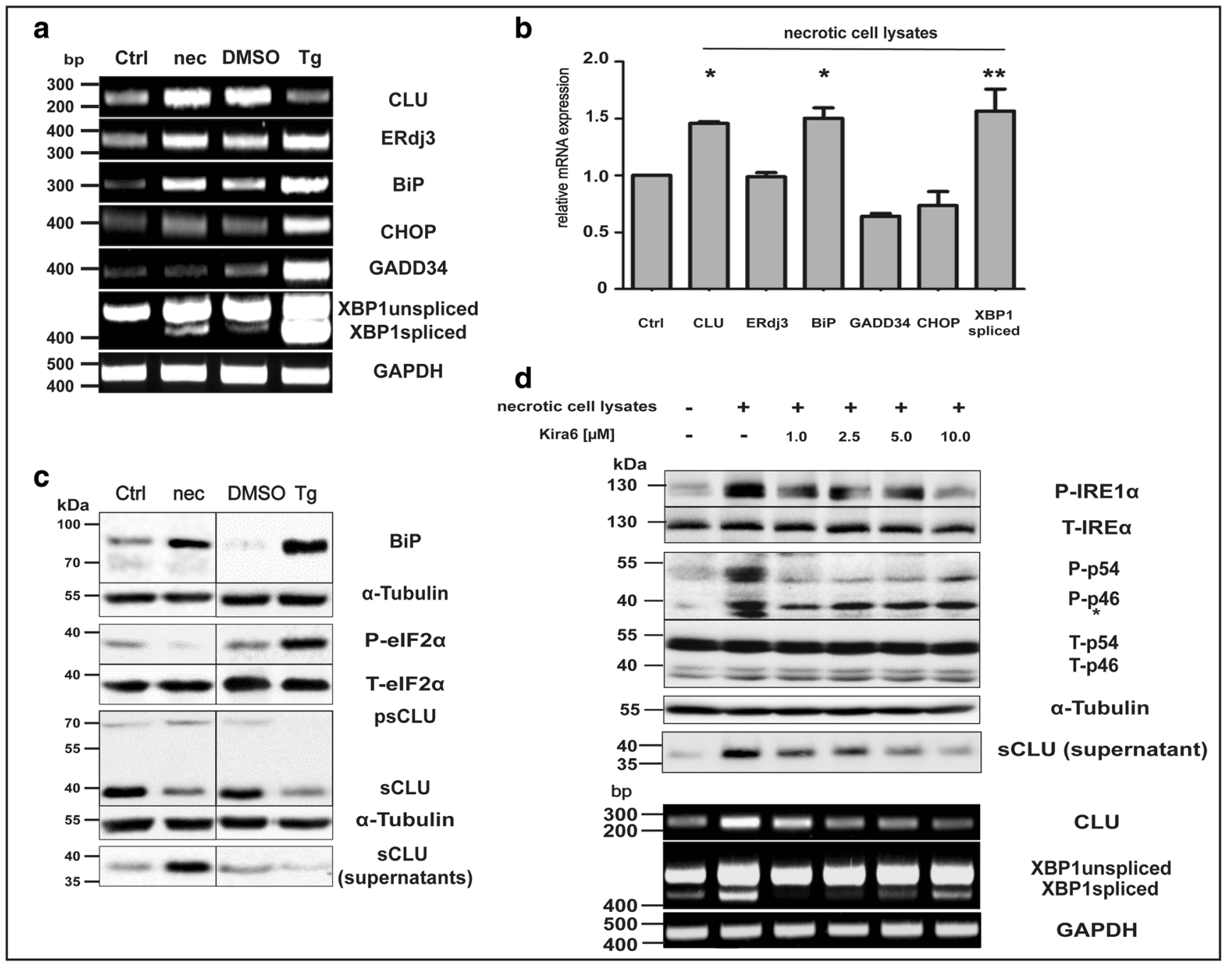

Fig. 3 Necrotic cell lysates induce the IRE1 $\alpha$ branch of the unfolded protein response (UPR). a RT-PCR analyses for the transcription of CLU, ERdj3, BiP, CHOP, GADD34, and XBP1 with GAPDH as loading control were performed in HEK-293 cells stimulated with $1 \mathrm{mg} / \mathrm{mL}$ necrotic cell lysates (nec) or $1 \mu \mathrm{M}$ thapsigargin $(\mathrm{Tg})$ for $6 \mathrm{~h}(n=3)$. b qRT-PCR analyses of HEK-293 cells stimulated with $1 \mathrm{mg} / \mathrm{mL}$ necrotic cell lysates were carried out. Values are represented as mean \pm SE from three independent experiments. Data were analyzed by one-way ANOVA, followed by Dunnett's test for multiple comparison $(* * * P<0.001 ; * * P<0.01$; $* P<0.05)$. c Western blot analyses of HEK-293 cells stimulated with $1 \mathrm{mg} / \mathrm{mL}$ necrotic cell lysates (nec) or $1 \mu \mathrm{M}$ thapsigargin (Tg) for $6 \mathrm{~h}$ (BiP and CLU) or $2 \mathrm{~h}(\mathrm{eIF} 2 \alpha)(n=3)$. d HEK-293 cells were preincubated in the presence of various concentrations of the IRE $1 \alpha$ kinase inhibitor Kira6 and subsequently stimulated with $1 \mathrm{mg} / \mathrm{mL}$ necrotic cell lysates in the presence of Kira6 for $2 \mathrm{~h}$ (Western blot of cell lysates) or $6 \mathrm{~h}$ (RT-PCR and Western blots of cell culture supernatants) $(n=3)$ 


\section{Results}

\section{Exposure of vital cells to necrotic cell lysates triggers JNK phosphorylation and a heat shock response}

Since an upregulation of CLU (APOJ) has been observed in cells challenged with necrotic cell lysates (Bach et al. 2001), we began using this gene to study the cellular response towards these exogenous stress conditions. At first, we focused on putative signaling pathways that have been described to upregulate CLU, such as the JNK/SAPK and the NF-KB signaling pathway, as well as the contribution of a heat shock response (Prochnow et al. 2013; Rohne et al. 2016; Viard et al. 1999; Woody and Zhao 2016).

Following exposure of HEK-293 cells to necrotic cell lysates, we found an elevation in CLU transcription and secretion (Fig. 1a and b). The increase in transcriptional upregulation and secretion was absent in cells treated with BSA, TNF, LPS, or FBS demonstrating the specificity of the cellular response (Fig. 1a and b). The transcriptional upregulation of CLU was abolished in the presence of the JNK inhibitor SP600125, indicating that JNK signaling is induced as a cellular response to necrotic cell lysates (Fig. 1c). As confirmed by Western blot analyses, the synthesis of CLU correlates to the augmented presence of phosphorylated JNK isoforms p46 and p54 which-like CLU expression - were impaired after application of the JNK inhibitor SP600125 (Fig. 2a). Comparable results were obtained after treatment with the proteasome inhibitor MG-132, a potent JNK inducer (Fig. 1c, Fig. 2a). Furthermore, by using AP-1 luciferase reporter assays, we demonstrated an increase in AP-1 promoter activity upon stimulation with necrotic cell
Fig. 4 Necrotic cell lysates elevate proliferation. a FACS analyses of cells stimulated for $24 \mathrm{~h}$ with necrotic cell lysates (nec) were performed using annexin V (apoptosis) or 7-AAD (necrosis) $(n=4)$. b Assessment of the ratio of vital cells, early apoptosis, and whole cell number (after $24 \mathrm{~h}$ of stimulation with $1 \mathrm{mg} / \mathrm{mL}$ necrotic cell lysates). Values shown are the mean $\pm \mathrm{SE}$ of three to four independent experiments. Data were analyzed by using an unpaired, two-tailed Student's $t$ test $(* * * P<0.001$; $* * P<0.01 ; * P<0.05)$. c 100,000 HEK-293 cells were seeded into 24-well plates and grow for $24 \mathrm{~h}$, subsequently the cells were stimulated with $1 \mathrm{mg} / \mathrm{mL}$ necrotic cell lysates (nec) for another $24 \mathrm{~h}$ and finally counted with a Neubauer counting chamber. Values shown are the mean $\pm \mathrm{SE}$ of seven independent experiments. Data were analyzed by using an unpaired, one-tailed Student's $t$ test $(* * * P<0.001 ; * * P<0.01$; $* P<0.05)$. MTS (d) and EdUClick assays (e) with HEK-293 cells after $24 \mathrm{~h}$ of stimulation with $1 \mathrm{mg} / \mathrm{mL}$ necrotic cell lysates (nec). Values shown are the mean \pm SE of three (d) to six (e) independent experiments. Data were analyzed by using an unpaired, two-tailed Student's $t$ test $(* * * P<0.001 ; * * P<0.01$; $* P<0.05)$
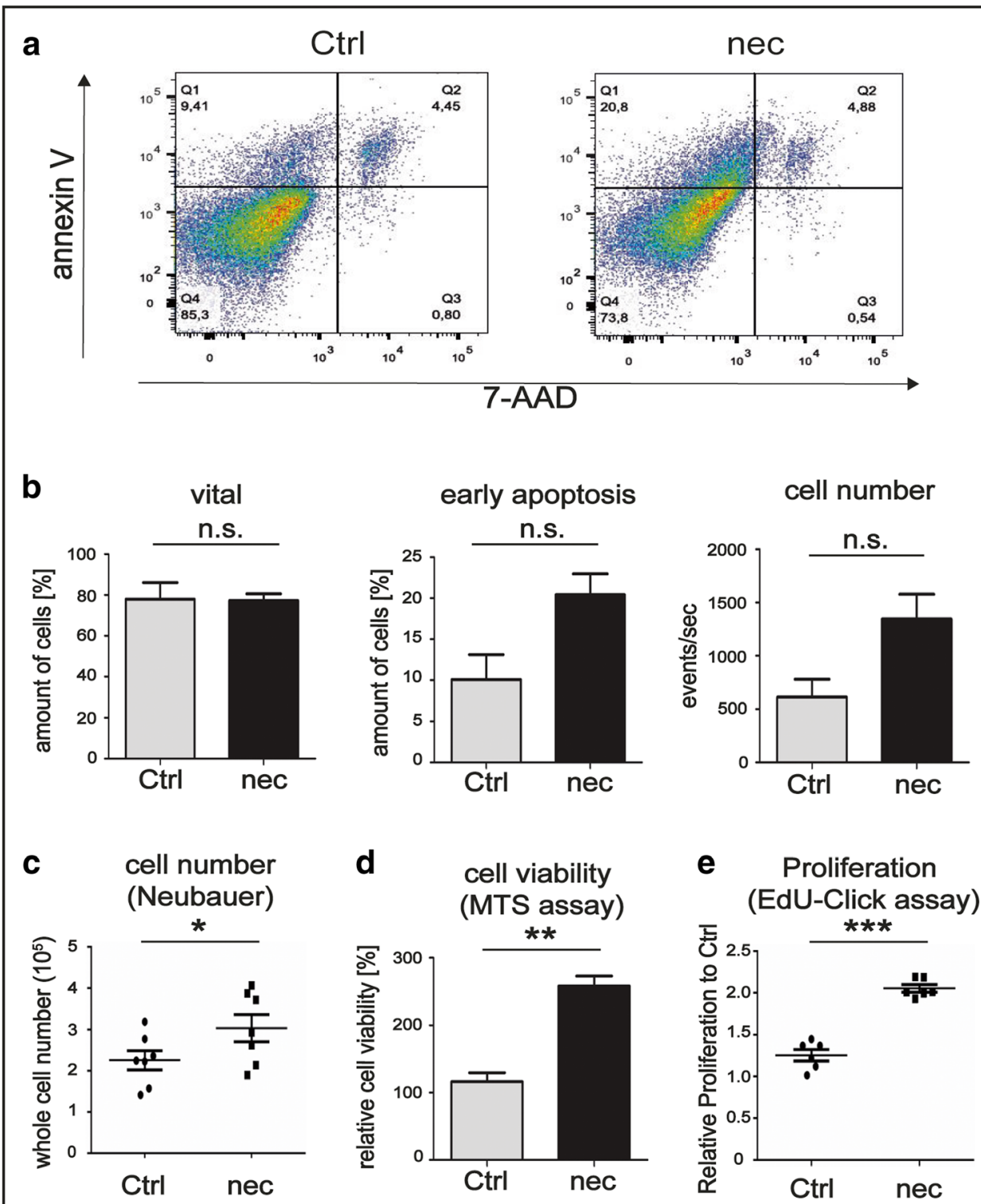
lysates, which was nearly abolished after application of SP600125 (Fig. 2b).

Together these results imply an involvement of the JNK/ AP-1 signaling pathway in the cellular response triggered by necrotic cell lysates.

Since a heat shock response can be induced by necrotic stress and other stresses apart from heat stress (Bromberg et al. 2013; Donati et al. 1990), we also tested the expression of the heat shock protein HSP27. As shown in Fig. $1 \mathrm{c}$, necrotic cell lysates as well as MG-132 stimulated the expression of HSP27 which was inhibited by SP 600125 suggesting that the necrotic cell lysate-triggered heat shock response is controlled by the JNK signaling pathway.
Encouraged by the recent findings of NF-KB-mediated induction of CLU in necrosis-exposed prostate cancer cells (Zhou et al. 2015), we studied the involvement of NF-KB in our experimental model. However, no involvement of the NF- $K B$ signaling pathway was observed after using the NF- $\mathrm{KB}$ inhibitor Parthenolide or by probing psCLU (presecretory clusterin) as well as sCLU (secretory clusterin) synthesis in NF-kB promoter assays (Fig. 2c and d).

\section{Necrotic cell lysates specifically induce IRE1 $\alpha$ phosphorylation and XBP1 splicing}

After having excluded Src kinase activation (Holzer et al. 2011) (Fig. 2e), nonspecific protein effects or TNF receptor
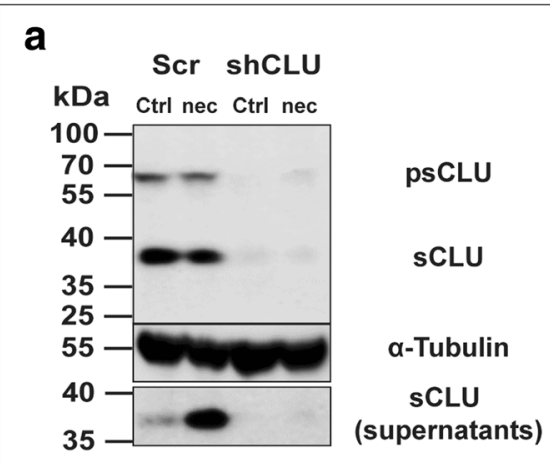

C

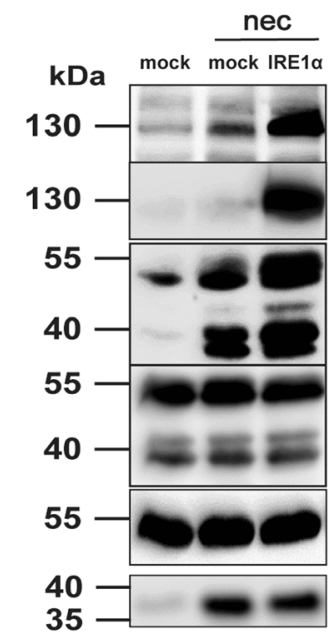

P-IRE1व

T-IRE $\alpha$

P-p54

P-p46

T-p54

T-p46

a-Tubulin

sCLU

(supernatants)
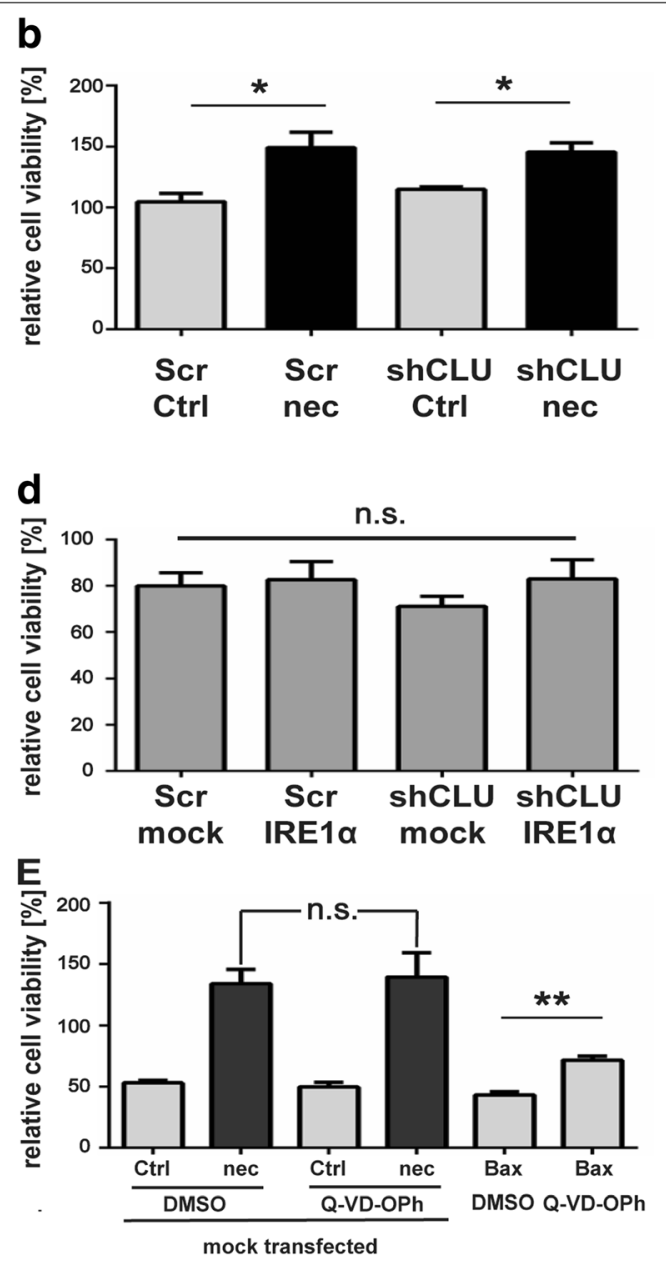

Fig. 5 CLU, IRE1 $\alpha$, and AiP does not contribute to NiP. (a and b) HEK293 cells stable transfected with pTER-EGFP encoding CLU shRNA (shCLU) or scramble (Scr) stimulated with $1 \mathrm{mg} / \mathrm{mL}$ necrotic cell lysates (nec) for $6 \mathrm{~h}$ (Western blot analyses, a) or $12 \mathrm{~h}$ (MTS assays, b). c HEK293 cells were transfected with pcDNA6 (mock) or IRE1 $\alpha$-pcDNA6 (IRE1 $\alpha$ ) for $6 \mathrm{~h}$, passaged to 6 -well plates and grown to full confluency. Subsequently, after $4 \mathrm{~h}$ of serum deprivation, the cells were stimulated with $0.5 \mathrm{mg} / \mathrm{mL}$ necrotic cell lysates (nec) for $2 \mathrm{~h}(n=4)$. d 10,000 HEK293 cells stable transfected with shCLU or Scr (see above) were seeded into 96-well plates and grown for $24 \mathrm{~h}$. Afterwards, the cells were transfected with pcDNA6 (mock) or IRE1 $\alpha$-pcDNA6 (IRE1 $\alpha$ ) (see above) for $6 \mathrm{~h}$. Subsequently, the cells were set serum-free for $12 \mathrm{~h}$ and finally MTS assays were performed. e 10,000 HEK-293 cells were seeded into 96-well plates and grown for $24 \mathrm{~h}$. Subsequently, the cells were transfected with pcDNA6 (mock) or BAX-pcDNA6 (BAX) (see above) for $18 \mathrm{~h}$ with concomitant stimulation of $1 \mathrm{mg} / \mathrm{mL}$ necrotic cell lysates for $12 \mathrm{~h}$ in the presence of $5 \mu \mathrm{M}$ Q-VD-OPh. All values shown are the mean \pm SE of three independent experiments. Data were analyzed by using an unpaired, two-tailed Student's $t$ test $(* * * P<0.001$; $* * P<0.01 ; * P<0.05)$ 
signaling (Fig. 1a, Fig. 2c) as activators, we tested if the exposure of vital cells to necrotic cell lysates would stimulate the unfolded protein response (UPR). If this were the case, JNK phosphorylation would be triggered by the IRE $1 \alpha$ branch of UPR via the TRAF2 adapter protein (Ron and Walter 2007).

Indeed, after $6 \mathrm{~h}$ of stimulation with necrotic cell lysates, we found significant XBP1 splicing and transcriptional upregulation of CLU and BiP, a key chaperone in the UPR. In contrast, ERdj3 (ATF6 branch), GADD34, and CHOP (PERK branch) were not induced (Fig. 3a and b). Along these lines, we also detected a translational upregulation of $\mathrm{BiP}$ (Fig. 3c), an enhancement of sCLU secretion, but no eIF $2 \alpha$ phosphorylation (PERK branch) (Fig. 3c) strengthening the notion that necrotic cell lysates specifically activate the IRE $1 \alpha$ branch of the UPR.

In contrast to necrotic cell lysates, the ER stress inducer thapsigargin activated the IRE $1 \alpha$ and the PERK branch of the UPR as demonstrated by the enhanced XBP1 splicing and elevated transcript levels of CHOP, GADD34 (Fig. 3a), as well as an increased BiP expression (Fig. 3a and c), and eIF $2 \alpha$ phosphorylation (Fig. 2c). Interestingly, after using the validated IRE1 $\alpha$ kinase inhibitor Kira6 (Ghosh et al. 2014) the transcriptional upregulation of CLU and secretion of sCLU was impaired in a dose-dependent manner (Fig. 3D). Concomitantly, JNK and IRE1 $\alpha$ phosphorylation also declined indicating a link of the UPR and CLU expression.

\section{Exposure of vital cells to necrotic cell lysates increases cell viability and proliferation}

Since JNK phosphorylation is known to initiate apoptotic cell death (Hetz 2012), we next determined whether cells treated with necrotic cell lysates exhibited an elevated level of apoptosis. Surprisingly, there was no significant difference in the amount of vital cells stimulated with necrotic cell lysates compared to control cells in FACS analyses using annexin Vand 7-AAD (Fig. 4a). Intriguingly, we even found an elevated number of cells in samples stimulated with necrotic cell lysates (Fig. $4 \mathrm{~b}$ and c). This result was supported by a 2.5 -fold increase in metabolic activity measured by MTS assays (Fig. 4d) and 1.7-fold increase in cell proliferation assays using the EdU-Click system (Fig. 4e).

CLU and IRE $1 \alpha$ are known to exert cytoprotective and even proliferative functions (Lin et al. 2007; Nguan et al. 2014; Rohne et al. 2016; Xu et al. 2014). Furthermore, it is known that caspase 3-mediated apoptosis can trigger cell proliferation in neighboring cells, a mechanism which is known as apoptosis-induced proliferation (AiP) (Huang et al. 2011; Ryoo et al. 2004). To test whether CLU, IRE1 $\alpha$, and/or AiP contribute to the observed proliferation, we used stable CLU knockdowns (Fig. 5a and b), IRE1 $\alpha$ overexpressing constructs (Fig. 5c and d), and the pan caspase inhibitor Q-VDOPh (Fig. 5e). In none of the approaches a modulation in necrotic cell lysate-mediated cell viability was observable pointing to an alternative mechanism.
This result prompted us to further elucidate signaling pathway(s) induced by necrotic cell lysates that might contribute to proliferation and viability. Since MAPK/ERK1/2 and mTOR signaling are known to stimulate cell proliferation (Mendoza et al. 2011), we tested the activation of these pathways. In cells treated with necrotic cell lysates, we found elevated levels of phosphorylated ERK1/2, mTOR, p70 and p85S6 kinase, and S6 ribosomal protein (Fig. 6a). Taken together, these data indicate that necrotic cell lysates promote cell proliferation and viability by activating MAPK/ERK1/2 and mTOR signal transduction pathways in vital cells.

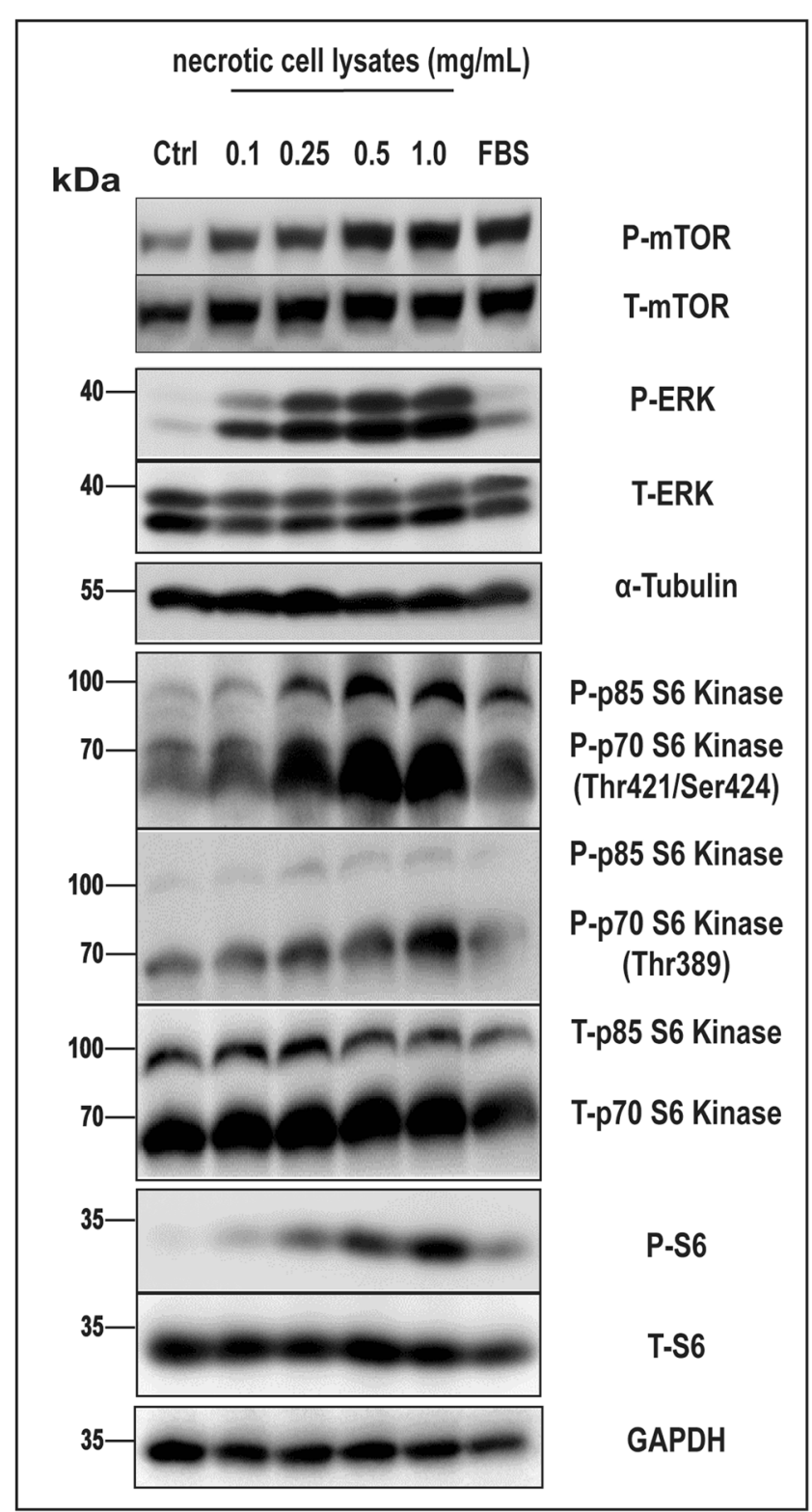

Fig. $6 \mathrm{MAPK} / \mathrm{ERK} 1 / 2$ and the mTOR signaling pathways are induced by necrotic cell lysates. HEK-293 cells were incubated with various concentrations of necrotic cell lysates $(\mathrm{mg} / \mathrm{mL})$ for $2 \mathrm{~h}$ and Western blots were performed $(n=3)$ 


\section{Discussion}

For years sitting on the "back shelf" of cell death research, necrotic cell death now emerges demonstrating to be a more and more coordinated and sophisticated cell death mechanism with a huge potential in biomedical research (Vanden Berghe et al. 2014). Involved in this setting is the extracellular chaperone clusterin (CLU), also known as apolipoprotein $\mathrm{J}$ (APOJ), which is highly correlated with necrosis, tissue degeneration, and apoptosis (Rohne et al. 2016; Woody and Zhao 2016). CLU is found to exert a cytoprotective role by helping to heal damaged tissues, clear the extracellular space from apoptotic cells, protein aggregates, and cellular debris (Baiersdorfer et al. 2010; Bartl et al. 2001; Cunin et al. 2016; Lee et al. 2011; Viard et al. 1999; Wilson and Zoubeidi 2016). To find a regulatory link between these two research fields, we decided to characterize the response mechanisms in vital cells to ongoing tissue necrosis. As a model system, we used necrotic cell lysates obtained from freeze/ thawed HEK-293 cells and applied it to vital HEK-293 cells. As observed in vivo we could demonstrate CLU transcriptional upregulation in this model (Fig. 1a). We further found an activation of the JNK/SAPK signaling pathway as well as a heat shock response and evidence for the involvement of these

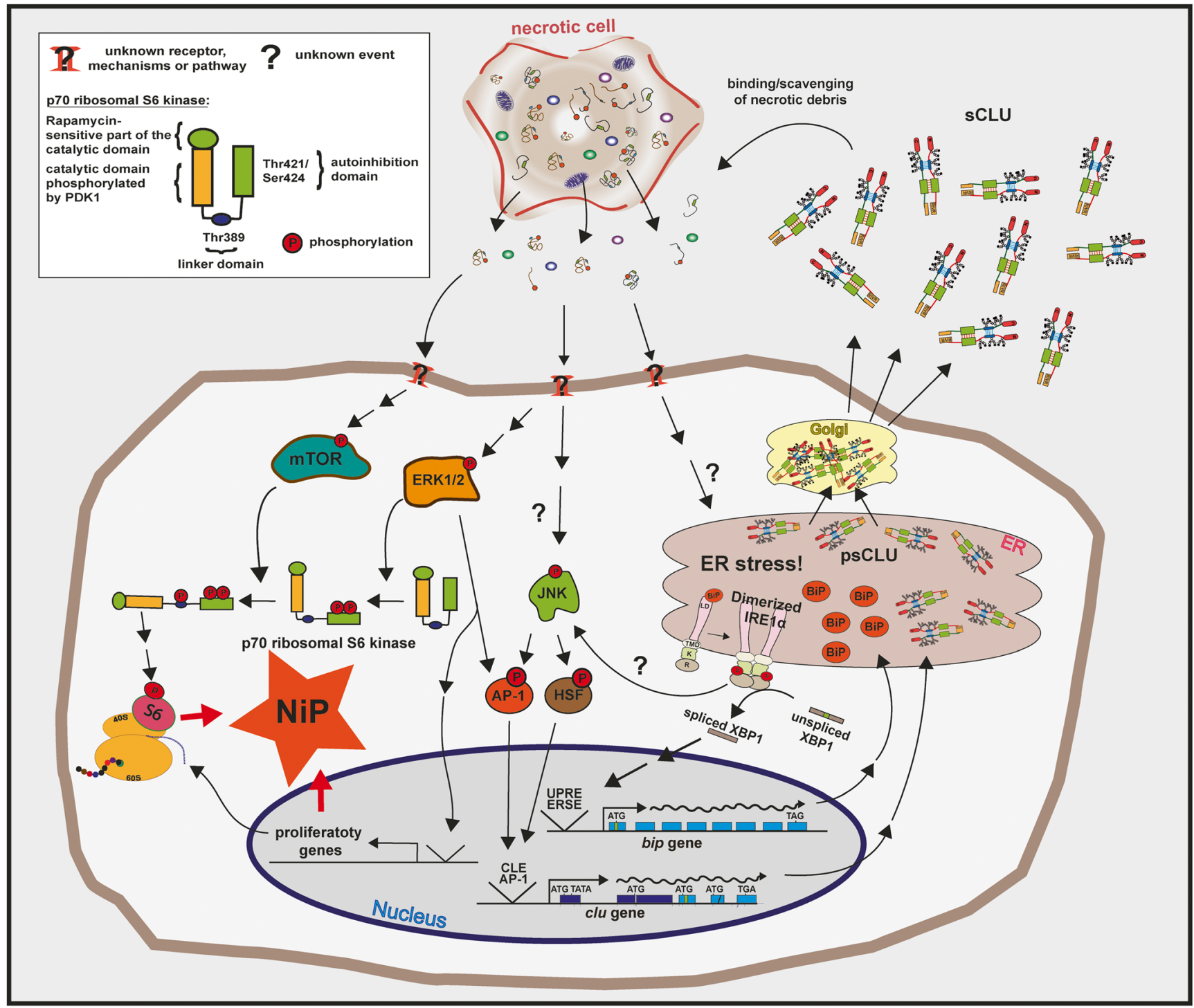

Fig. 7 Signal transduction pathways induced by necrotic cell lysates in HEK-293 cells. Lysates released from necrotic cells induce the MAPK/ERK1/2, mTOR, JNK, and IRE1 $\alpha$ signaling pathways. Following ERK1/2 and mTOR signaling, the cell viability and proliferation is enhanced due to increased protein translation promoted by phosphorylated S6 ribosomal protein (among others), a mechanism which is called NiP (Necrosis-induced Proliferation). The JNK and IRE1 $\alpha$ signaling pathways are inducing the expression of intra - and extracellular chaperones such as BiP (mediated by XBP1) and CLU (mediated by AP-1, supported by a concomitant heat shock response, HSF). Eventually, the cell releases secretory clusterin (sCLU) thus facilitating the removal of cellular debris (Bartl et al. 2001; Rohne et al. 2014) 
pathways in CLU upregulation by using the JNK inhibitor SP600125 (Fig. 1a, Fig. 2a and b). An involvement of NF- $\mathrm{KB}$ as seen in similar experiments by Zhou et al. 2015 could not be confirmed in our experimental model (Fig. 2c and d).

Furthermore, we could demonstrate that cells stimulated with necrotic cell lysates suffer ER stress which results in the specific induction of the IRE1 $\alpha$ branch of the UPR (Fig. $3 \mathrm{a}, \mathrm{b}$, and d). Intriguingly, it was shown by Alnasser et al. 2016 that CLU might be part of this cellular response and therefore involved in a sophisticated cellular chaperone network, the "chaperome" (Finka and Goloubinoff 2013). On the basis of decreased IRE1 $\alpha$ phosphorylation in CLU knockout cells under ischemic conditions, they proposed that CLU might act as a supporting chaperone in the UPR. Indeed, by using the IRE1 $\alpha$ kinase inhibitor Kira6 we have found a decline in CLU expression (Fig. 3d). More recently, it was suggested that severe ER stress induced by thapsigargin abolishes CLU upregulation and prevents its secretion (Genereux et al. 2015; Nizard et al. 2007), a finding that was also supported in our experimental model (Fig. 3a and c). We therefore argue that CLU expression and secretion can only be present or promoted under ER stress encompassing solely the IRE $1 \alpha$ branch (see also Fig. 5c), but is inhibited by the induction of the PERK branch.

However, by testing whether necrotic stress causes increased apoptosis, revealed that the cell number and viability was enhanced (1.5-fold within $12 \mathrm{~h}$ and up to 2.5 -fold within 24 h; Fig. 5b and Fig. 4d). No significant apoptosis was detectable (Fig. 4a and b). Moreover, it was measured that the cells indeed start to proliferate (Fig. 4e). Important pathways stimulating cell proliferation are the MAPK/ERK1/2 and the mTOR signaling pathways (Mendoza et al. 2011). We show that necrotic cell lysates induce the ERK1/2 and mTOR signaling pathways, whereas the FBS control solely induces the mTOR signaling pathway (Fig. 6). Additionally, concurrent with mTOR phosphorylation, the phosphorylation of p70S6 kinase at position Thr389 and S6 is enhanced (Fig. 6). ERK1/ 2 phosphorylates the residues Thr421/Ser424 at the autoinhibitory domain of the p70S6 kinase to make the kinase accessible for mTOR-mediated phosphorylation at position Thr389 which serves as a platform for PDK1 for full activation of the p70S6 kinase and subsequent S6 phosphorylation (Fig. 6 and Fig. 7) (Lehman et al. 2003; Pullen and Thomas 1997). The phosphorylation of ERK1/2, however, occurs already at low concentrations of necrotic cell lysates and is accompanied by Thr421/Ser424 phosphorylation of the p70S6 kinase (Fig. 6). This suggests a decisive role for ERK1/2 in the mTOR/p70S6 kinase/S6 signaling pathway.

It was recently shown in drosophila and in human cancer cells that apoptotic cells release growth stimulating signals into the extracellular fluid resulting in repopulation, a process that might be crucial to tissue reconstruction and remodeling, but is harmful when occurring during tumor therapy (Huang et al. 2011; Ryoo et al. 2004). To our knowledge, it has up to now never been considered whether cells undergoing necrotic cell death can likewise directly promote proliferation of surrounding cells. The fact that the MAPK/ERK1/2 and mTOR signal transduction pathways are activated immediately after application of necrotic cell lysates implies that necrotic cells release mitogenic signaling molecules directly into the extracellular space that exert the proliferative effect. Furthermore, inhibiting apoptosis with the pan caspase inhibitor Q-VD-OPh did not suppress the induction of cell proliferation demonstrating that it is mediate by components of the necrotic cell lysate rather than being a secondary effect initiated by apoptotic cells. To describe this process, we propose the term: Necrosis-induced Proliferation (NiP). For the first time, we show that the induction of proliferation of vital surrounding cells initiated by dying neighboring cells is not restricted to caspase 3-dependent apoptosis as primary stimulus (Fig. 5e). An overview is depicted in Fig. 7.

In summary, our results broaden our knowledge on compensatory proliferation induced by apoptotic cells to cells undergoing accidental cell death and therefore have the same profound implications for the therapy of tumors.

Acknowledgements We like to thank Georgiana Maria Klug-Micu for the generation and selection of the stably transfected shCLU and Scr HEK-293 cell lines.

\section{References}

Alnasser HA, Guan Q, Zhang F, Gleave ME, Nguan CY, Du C (2016) Requirement of clusterin expression for prosurvival autophagy in hypoxic kidney tubular epithelial cells. Am J Physiol Renal Physiol 310:F160-F173. doi:10.1152/ajprenal.00304.2015

Bach UC, Baiersdorfer M, Klock G, Cattaruzza M, Post A, Koch-Brandt C (2001) Apoptotic cell debris and phosphatidylserine-containing lipid vesicles induce apolipoprotein. J (clusterin) gene expression in vital fibroblasts Experimental cell research 265:11-20. doi:10. 1006/excr.2001.5159

Baiersdorfer M et al (2010) Toll-like receptor 3 mediates expression of clusterin/apolipoprotein. J in vascular smooth muscle cells stimulated with RNA released from necrotic cells experimental cell research 316:3489-3500. doi:10.1016/j.yexcr.2010.07.022

Bartl MM, Luckenbach T, Bergner O, Ullrich O, Koch-Brandt C (2001) Multiple receptors mediate apoJ-dependent clearance of cellular debris into nonprofessional phagocytes. Exp Cell Res 271:130-141. doi:10.1006/excr.2001.5358

Bauskar A et al (2015) Clusterin seals the ocular surface barrier in mouse dry eye. PLoS One 10:e138958. doi:10.1371/journal.pone. 0138958

Bromberg Z, Goloubinoff P, Saidi Y, Weiss YG (2013) The membraneassociated transient receptor potential vanilloid channel is the central heat shock receptor controlling the cellular heat shock response in epithelial cells. PLoS One 8:e57149. doi:10.1371/journal.pone. 0057149 
Cunin P et al (2016) Clusterin facilitates apoptotic cell clearance and prevents apoptotic cell-induced autoimmune responses. Cell Death Dis 7:e2215. doi:10.1038/cddis.2016.113

Donati YR, Slosman DO, Polla BS (1990) Oxidative injury and the heat shock response. Biochem Pharmacol 40:2571-2577

Festjens N, Vanden Berghe T, Vandenabeele P (2006) Necrosis, a wellorchestrated form of cell demise: signalling cascades, important mediators and concomitant immune response. Biochim Biophys Acta 1757:1371-1387. doi:10.1016/j.bbabio.2006.06.014

Finka A, Goloubinoff P (2013) Proteomic data from human cell cultures refine mechanisms of chaperone-mediated protein homeostasis. Cell Stress Chaperones 18:591-605. doi:10.1007/s12192-013-0413-3

Gardner BM, Pincus D, Gotthardt K, Gallagher CM, Walter P (2013) Endoplasmic reticulum stress sensing in the unfolded protein response. Cold Spring Harb Perspect Biol 5:a013169. doi:10.1101/ cshperspect.a013169

Genereux JC et al (2015) Unfolded protein response-induced ERdj3 secretion links ER stress to extracellular proteostasis. EMBO J 34:4 19. doi:10.15252/embj.201488896

Ghosh R et al (2014) Allosteric inhibition of the IRE1alpha RNase preserves cell viability and function during endoplasmic reticulum stress. Cell 158:534-548. doi:10.1016/j.cell.2014.07.002

Hetz C (2012) The unfolded protein response: controlling cell fate decisions under ER stress and beyond. Nat Rev Mol Cell Biol 13:89102. doi:10.1038/nrm3270

Hirota M, Kitagaki M, Itagaki H, Aiba S (2006) Quantitative measurement of spliced XBP1 mRNA as an indicator of endoplasmic reticulum stress. J Toxicol Sci 31:149-156

Holzer RG et al (2011) Saturated fatty acids induce c-Src clustering within membrane subdomains, leading to JNK activation. Cell 147:173184. doi:10.1016/j.cell.2011.08.034

Huang Q et al (2011) Caspase 3-mediated stimulation of tumor cell repopulation during cancer radiotherapy. Nat Med 17:860-866. doi: $10.1038 / \mathrm{nm} .2385$

Kalyanaraman B (2013) Teaching the basics of redox biology to medical and graduate students: oxidants, antioxidants and disease mechanisms. Redox Biol 1:244-257. doi:10.1016/j.redox.2013.01.014

Lee $S$ et al (2011) Essential role of clusterin in pancreas regeneration. Dev Dyn 240:605-615. doi:10.1002/dvdy.22556

Leeb C, Eresheim C, Nimpf J (2014) Clusterin is a ligand for apolipoprotein $\mathrm{E}$ receptor 2 (ApoER2) and very low density lipoprotein receptor (VLDLR) and signals via the reelin-signaling pathway. J Biol Chem 289:4161-4172. doi:10.1074/jbc.M113.529271

Leek RD, Landers RJ, Harris AL, Lewis CE (1999) Necrosis correlates with high vascular density and focal macrophage infiltration in invasive carcinoma of the breast. Br J Cancer 79:991-995. doi:10. 1038/sj.bjc. 6690158

Lehman JA, Calvo V, Gomez-Cambronero J (2003) Mechanism of ribosomal p70S6 kinase activation by granulocyte macrophage colonystimulating factor in neutrophils: cooperation of a MEK-related, THR421/SER424 kinase and a rapamycin-sensitive, m-TORrelated THR389 kinase. J Biol Chem 278:28130-28138. doi:10. 1074/jbc.M300376200

Li N, Zoubeidi A, Beraldi E, Gleave ME (2013) GRP78 regulates clusterin stability, retrotranslocation and mitochondrial localization under ER stress in prostate cancer. Oncogene 32:1933-1942. doi:10. 1038/onc.2012.212

Lin JH et al (2007) IRE1 signaling affects cell fate during the unfolded protein response. Science 318:944-949. doi:10.1126/science. 1146361

Martinet W, Schrijvers DM, De Meyer GR (2011) Necrotic cell death in atherosclerosis. Basic Res Cardiol 106:749-760. doi:10.1007/ s00395-011-0192-x

McLaughlin L et al (2000) Apolipoprotein J/clusterin limits the severity of murine autoimmune myocarditis. J Clin Invest 106:1105-1113. doi:10.1172/JCI9037
Mendoza MC, Er EE, Blenis J (2011) The Ras-ERK and PI3K-mTOR pathways: cross-talk and compensation trends. Biochem Sci 36: 320-328. doi:10.1016/j.tibs.2011.03.006

Merlotti A et al (2015) Fucosylated clusterin in semen promotes the uptake of stress-damaged proteins by dendritic cells via DC-SIGN. Hum Reprod 30:1545-1556. doi:10.1093/humrep/dev113

Michel D, Chatelain G, North S, Brun G (1997) Stress-induced transcription of the clusterin/apoJ gene. The Biochemical journal 328( $\mathrm{Pt} \mathrm{1)}$ : $45-50$

Nguan CY, Guan Q, Gleave ME, Du C (2014) Promotion of cell proliferation by clusterin in the renal tissue repair phase after ischemiareperfusion injury. Am J Physiol Renal Physiol 306:F724-F733. doi:10.1152/ajprenal.00410.2013

Nizard P, Tetley S, Le Drean Y, Watrin T, Le Goff P, Wilson MR, Michel D (2007) Stress-induced retrotranslocation of clusterin/apoJ into the cytosol. Traffic 8:554-565. doi:10.1111/j.1600-0854.2007.00549.x

Prochnow H, Gollan R, Rohne P, Hassemer M, Koch-Brandt C, Baiersdorfer M (2013) Non-secreted clusterin isoforms are translated in rare amounts from distinct human mRNA variants and do not affect Bax-mediated apoptosis or the NF-kappaB signaling pathway. PLoS One 8:e75303. doi:10.1371/journal.pone.0075303

Pullen N, Thomas G (1997) The modular phosphorylation and activation of p70s6k. FEBS Lett 410:78-82

Rohne P, Prochnow H, Wolf S, Renner B, Koch-Brandt C (2014) The chaperone activity of clusterin is dependent on glycosylation and redox environment. Cellular physiology and biochemistry : international journal of experimental cellular physiology, biochemistry, and pharmacology 34:1626-1639. doi:10.1159/000366365

Rohne P, Prochnow H, Koch-Brandt C (2016) The CLU-files: disentanglement of a mystery. Biomolecular concepts 7:1-15. doi:10.1515/ bmc-2015-0026

Ron D, Walter P (2007) Signal integration in the endoplasmic reticulum unfolded protein response. Nat Rev Mol Cell Biol 8:519-529. doi: $10.1038 / \mathrm{nrm} 2199$

Ryoo HD, Gorenc T, Steller H (2004) Apoptotic cells can induce compensatory cell proliferation through the JNK and the wingless signaling pathways. Dev Cell 7:491-501. doi:10.1016/j.devcel.2004. 08.019

Sabatte J et al (2011) Semen clusterin is a novel DC-SIGN ligand. J Immunol 187:5299-5309. doi:10.4049/jimmunol.1101889

Schwarz M et al (2008) Potential protective role of apoprotein J (clusterin) in atherogenesis: binding to enzymatically modified low-density lipoprotein reduces fatty acid-mediated cytotoxicity. Thromb Haemost 100:110-118. doi:10.1160/TH07-12-0737

Sidrauski C et al (2013) Pharmacological brake-release of mRNA translation enhances cognitive memory. elife 2:e00498. doi:10.7554/ eLife.00498

Srikrishna G, Freeze HH (2009) Endogenous damage-associated molecular pattern molecules at the crossroads of inflammation and cancer. Neoplasia 11:615-628

Troakes C, Smyth R, Noor F, Maekawa S, Killick R, King A, Al-Sarraj S (2016) Clusterin expression is upregulated following acute head injury and localizes to astrocytes in old head injury. Neuropathology. doi:10.1111/neup. 12320

Vakkila J, Lotze MT (2004) Inflammation and necrosis promote tumour growth. Nat Rev Immunol 4:641-648. doi:10.1038/nri1415

van Schadewijk A, van't Wout EF, Stolk J, Hiemstra PS (2012) A quantitative method for detection of spliced X-box binding protein-1 (XBP1) mRNA as a measure of endoplasmic reticulum (ER) stress. Cell Stress Chaperones 17:275-279. doi:10.1007/s12192-0110306-2

Vanden Berghe T, Linkermann A, Jouan-Lanhouet S, Walczak H, Vandenabeele P (2014) Regulated necrosis: the expanding network of non-apoptotic cell death pathways. Nat Rev Mol Cell Biol 15: 135-147. doi:10.1038/nrm3737 
Viard I et al (1999) Clusterin gene expression mediates resistance to apoptotic cell death induced by heat shock and oxidative stress. The Journal of investigative dermatology 112:290-296. doi:10. 1046/j.1523-1747.1999.00531.x

Wilson MR, Zoubeidi A (2016) Clusterin as a therapeutic target expert. Opin Ther Targets:1-13. doi:10.1080/14728222.2017.1267142

Woody SK, Zhao L (2016) Clusterin (APOJ) in Alzheimer's disease: an old molecule with a new role. Update on Dementia.

Wyatt AR, Yerbury JJ, Berghofer P, Greguric I, Katsifis A, Dobson CM, Wilson MR (2011) Clusterin facilitates in vivo clearance of extracellular misfolded proteins. Cellular and molecular life sciences : CMLS 68:3919-3931. doi:10.1007/s00018-011-0684-8)

Wyatt AR, Yerbury JJ, Dabbs RA, Wilson MR (2012) Roles of extracellular chaperones in amyloidosis. J Mol Biol 421:499-516. doi:10. 1016/j.jmb.2012.01.004

$\mathrm{Xu}$ T et al (2014) The IRE1alpha-XBP1 pathway regulates metabolic stress-induced compensatory proliferation of pancreatic beta-cells. Cell Res 24:1137-1140. doi:10.1038/cr.2014.55

Yan MM, Ni JD, Song D, Ding M, Huang J (2015) Interplay between unfolded protein response and autophagy promotes tumor drug resistance. Oncol Lett 10:1959-1969. doi:10.3892/ol.2015.3508
Yoshida H, Matsui T, Yamamoto A, Okada T, Mori K (2001) XBP1 mRNA is induced by ATF6 and spliced by IRE1 in response to ER stress to produce a highly active transcription factor. Cell 107: 881-891

Yuan J (2009) Neuroprotective strategies targeting apoptotic and necrotic cell death for stroke. Apoptosis 14:469-477. doi:10.1007/s10495008-0304-8

Zhang F et al (2014) Clusterin facilitates stress-induced lipidation of LC3 and autophagosome biogenesis to enhance cancer cell survival. Nat Commun 5:5775. doi:10.1038/ncomms6775

Zhou J, Chen X, Gilvary DL, Tejera MM, Eksioglu EA, Wei S, Djeu JY (2015) HMGB1 induction of clusterin creates a chemoresistant niche in human prostate tumor cells. Sci Rep 5:15085. doi:10. 1038/srep 15085

Zlokovic BV et al (1996) Glycoprotein 330/megalin: probable role in receptor-mediated transport of apolipoprotein $\mathrm{J}$ alone and in a complex with Alzheimer disease amyloid beta at the blood-brain and blood-cerebrospinal fluid barriers. Proc Natl Acad Sci U S A 93: $4229-4234$ 